\title{
Spiral-Wave Instability in a Medium with a Gradient in the Fibroblast Density: a Computational Study
}

\author{
Soling Zimik ${ }^{1}$, Rahul Pandit ${ }^{1}$ \\ ${ }^{1}$ Centre for Condensed Matter Theory, Department of Physics, Indian Institute of Science, \\ Bangalore, 560012, India
}

\begin{abstract}
Fibrosis, a process of fibroblast proliferation in cardiac tissue, is a major concern for patients with diseases like ischemia, heart failure, and cardiomyopathy, because of its arrhythmogenic effects. Fibroblasts, in appreciable densities, are known to affect the electrical-wave dynamics in cardiac tissue, because the coupling of fibroblasts with myocytes modulates the electrophysiological properties of the myocytes. Furthermore, in fibrotic hearts, the distribution of fibroblasts can be heterogeneous, so the density of fibroblasts can vary from wounded regions (like infarcted or ischemic zones) to normal regions of the heart. Such gradients in the fibroblast density (GFD) induce spatial variation in the electrophysiological properties of the tissue, and the latter can initiate and affect the dynamics of pathological waves like spiral waves. We study the effects of GFDs on the dynamics of spiral waves by using a state-of-theart mathematical model for human-ventricular tissue. We find that, in the presence of GFDs, spiral waves are unstable, i.e., a stable spiral wave breaks into multiple spiral waves. We find that GFDs induce spatial variations in the local spiral-wave frequency $\omega$ in the medium. Such a variation in $\omega$ leads to anisotropic thinning of the spiral arm that gives way to spiral-wave breaks. We also study the factors that enhance the instability of the spiral waves in the medium with GFD. Finally, we show that the presence of GFD can spontaneously lead to spiral waves, via highfrequency pacing, in the medium.
\end{abstract}

\section{Introduction}

The occurrence of abnormal spatiotemporal patterns of electrical waves in cardiac tissue, in the form of spiral waves, has been associated with cardiac arrhythmias [1]. The existence of a single spiral wave is associated with tachycardia [2], and a multiple-spiral-wave state is associated with a life-threatening arrhythmia known as fibrillation [3]. A single-spiral-wave state can transition into a multiple-spiral-wave state if the spiral becomes unsta- ble and breaks up, giving rise to many daughter spirals [1]. Given that fibrillation is a more lethal condition than tachycardia, it is important to understand the mechanisms of the transition process from a single- to a multiple-spiral-wave state. Here, we present a mechanism of spiral-wave instability in a medium with a gradient in fibroblast density (GFD).

Fibroblasts are passive cells that are needed for the proper functioning of a heart, because they, along with other cells, form the extracellular matrix and ensure the structural integrity of the heart. However, the abnormal proliferation of fibroblasts because of diseases like ischemia, heart failure, and cardiomyopathy is considered to be arrhythmogenic [4]. Fibroblasts, if they form gapjunctional coupling, can change the electrophysiology of myocytes in cardiac tissue [5]. This, in turn, can affect the dynamics of electrical waves in cardiac tissue. Many studies have been performed to investigate the effects of fibroblasts on wave dynamics in cardiac tissue [6,7]; however, most of the studies, which consider fibroblast-myocyte (FM) coupling, deal with a homogeneous distribution of fibroblasts in the domain. But, the density of fibroblasts in a diseased cardiac tissue may not necessarily be homogeneous [8]. Therefore, it is important to study the effects of FM coupling on wave dynamics in a domain with heterogeneous distributions of fibroblast density.

We investigate the effects of GFD on spiral-wave dynamics in a mathematical model for human-ventricular tissue. We find that GFD induces a spatial variation of the local spiral-wave frequency $\omega$ in the domain. This variation of $\omega$ in the domain leads to spiral-wave instability, and the degree of instability depends on the magnitude of the variation of $\omega$. We also investigate the factors that modulate the variation of $\omega$ in the domain. We find that, for a given GFD, the resting membrane potential $E_{f}$ of the fibroblasts, and the number of fibroblasts $N_{f}$ that are coupled to a myocyte can change the spatial variation of $\omega$ in the domain. Finally, we also show that GFD can spontaneously initiate spiral waves via high-frequency pacing. 


\section{Methods And Materials}

We use the O'Hara-Rudy dynamic model [9] for our human-ventricular myocyte cell. The fibroblasts are modelled as passive cells; for these fibroblasts, we use the model given by MacCannell, et al. [10].

In our two-dimensional (2D) simulations the fibroblasts are attached atop the myocytes; thus, our 2D simulation domain is a bilayer. The spatiotemporal evolution of the membrane potential $\left(\mathrm{V}_{\mathrm{m}}\right)$ of the myocytes in tissue is governed by a reaction-diffusion equation, which is the following partial-differential equation (PDE):

$$
\frac{\partial \mathrm{V}_{\mathrm{m}}}{\partial t}+\frac{I_{\text {ion }}+I_{\text {gap }}}{C_{m}}=\mathrm{D} \nabla^{2} \mathrm{~V}_{\mathrm{m}}
$$

where $\mathrm{D}$ is the diffusion constant between the myocytes. $I_{\text {ion }}$ is the sum of all the ionic currents of the myocyte [9]. $I_{\text {gap }}$ is the gap-junctional current between a myocyte and a fibroblast in an FM composite. $I_{\text {gap }}=0$ if no fibroblast is attached to a myocyte.

We use the forward-Euler method for time marching with a five-point stencil for the Laplacian. We set $\mathrm{D}=$ $0.0012 \mathrm{~cm}^{2} / \mathrm{ms}$. The temporal and spatial resolutions are set to be $\delta x=0.02 \mathrm{~cm}$ and $\delta t=0.02 \mathrm{~ms}$, respectively. We use a domain size of $960 \times 960$ grid points. We initiate the spiral wave by using the conventional S1-S2 cross-field protocol.

\section{Results}

The coupling of fibroblasts to a myocyte can change the electrophysiological properties of the myocyte. We show in fig. 1 the action potentials (APs) of a myocyte coupled to fibroblasts with different values of $E_{f}$ : The black curve indicates the AP of an isolated myocyte, and the red, and blue curves indicate the APs of a myocyte coupled to fibroblasts of $E_{f}=-25 \mathrm{mV}$, and $-15 \mathrm{mV}$, respectively. We can see that the action potential duration (APD) of the myocyte increases if it is coupled to fibroblasts, and the increase in the APD depends on the value of $E_{f}$ of the fibroblasts.

The changes in the APDs of myocytes because of fibroblast coupling can affect the electrical-wave properties in a tissue. For example, changes in the APD of the consituent myocytes affect the frequency $\omega$ of a spiral wave as follows. Consider a stably rotating spiral wave, if we neglect curvature effects, dimensional analysis gives $\omega \simeq \frac{\theta}{\lambda}$, where $\theta$ is the conduction velocity and $\lambda$ is the wavelength. Furthermore, $\lambda \simeq \theta \times \mathrm{APD}$, and, therefore,

$$
\omega \simeq \frac{1}{\mathrm{APD}}
$$

In a domain with, on average, a homogeneous distribution of fibroblasts (see fig. 2 (a)), the spiral-wave frequency $\omega$ decrease with the increase of fibroblast percentage $p_{f}$ (see fig. 3), because as fibroblast coupling increase

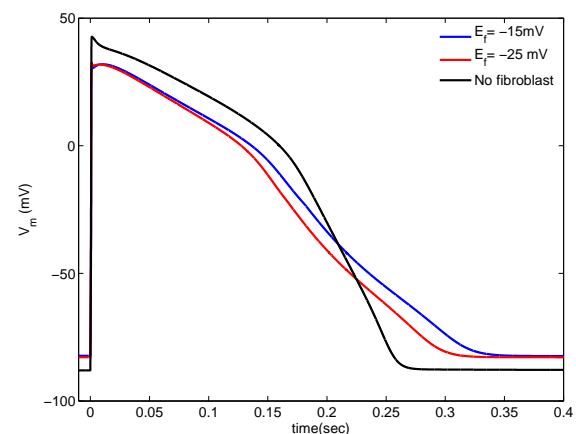

Figure 1. Plots showing the action potentials of an isolated myocyte (black curve), and a myocyte attached to $\mathrm{N}_{\mathrm{f}}=2$ fibroblasts of $E_{\mathrm{f}}=-15 \mathrm{mV}$ (blue curve), and $\mathrm{E}_{\mathrm{f}}=-25 \mathrm{mV}$ (red curve).

the APD of the myocytes (see fig. 1), the increase in $p_{f}$ increases, on average, the APD of the myocytes in the medium; and, thus, eqn. 2 implies that $\omega$ decreases with the increase of $p_{f}$. Figure 3 shows the variation of $\omega$ with $p_{f}$. (a)

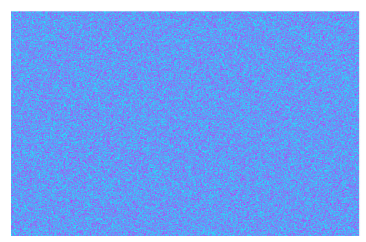

(b)

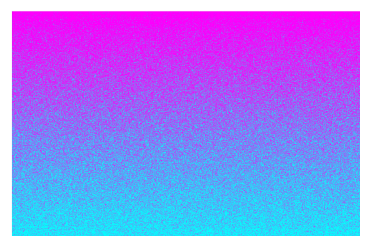

Figure 2. Pseudocolor plots showing two types of fibroblast distributions. (a) the density distribution of fibroblasts is uniform throughout the domain, on average. The cyan color indicates myocyte density with no fibroblasts, and the magenta color indicates fibroblast-myocyte composites. The value of $p_{f}$ is $40 \%$. (b) There is a gradient of fibroblast density (GFD) along the vertical y axis, where $p_{f}$ varies linearly from $10 \%$ (at the bottom of the domain) to $100 \%$ (at the top of the domain).

\subsection{Effects of GFD on the stability of spiral waves}

The presence of gradient in fibroblast density (GFD) induces spiral-wave instability. Figure 2 (b) shows the GFD in a domain, where the fibroblast density increases from the bottom $\left(p_{f}=10 \%\right)$ to the top $\left(p_{f}=100 \%\right)$ of the domain. The magenta and cyan colors indicate fibroblast-mycoyte composites and myocytes, respectively. Figure 4 shows the break-up of a spiral wave in the domain shown in fig. 2 (b). We see that the spiral wave breaks in the top region where the density of fibroblasts is high. The reason for the spiral- 
(a)
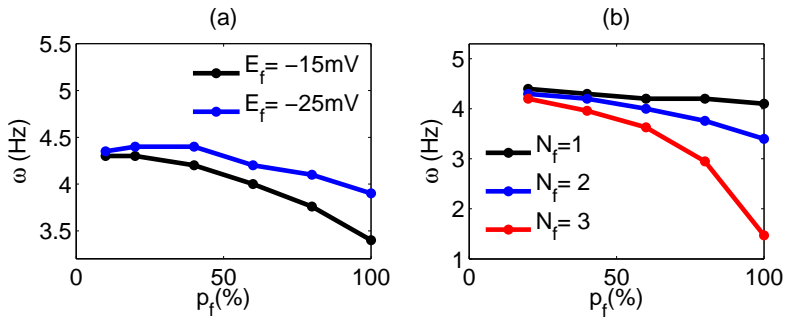

Figure 3. Variation of the spiral-wave frequency $\omega$ with the percentage of fibroblasts $p_{f}$, in a domain with a homogeneous fibroblast distribution. (a) Plots showing the variation of $\omega$ with $p_{f}$ for $\mathrm{E}_{\mathrm{f}}=-15 \mathrm{mV}$ (black curve), and $\mathrm{E}_{\mathrm{f}}=$ $-25 \mathrm{mV}$ (blue curve). (b) Plots showing the variation of $\omega$ with $p_{f}$ for three different values of $N_{f}=1$ (black curve), $N_{f}=2$ (blue curve), and $N_{f}=3$ (red curve).

wave instability is as follows. The presence of GFD in the medium induces a spatial variation of the local value of $\omega$ (refer fig. 3). In fig. 4 the local value of $\omega$ decreases from the bottom to the top of the domain. This anisotropic variation of $\omega$ leads to an anisotropic thinning of the wavelength of the spiral arms (see fig. $42.14 \mathrm{~s}$ ), which gives way for spiral-wave break-up. The wave-thinning occurs in the upper region, because it has large APD. As the upper region has a large APD, the conduction velocity is lower in that region (conduction-velocty restitution property [11]); therefore, in order to support the high-frequency wave trains coming from the lower region, the wavelength of the waves thins as it propagates towards the top region.

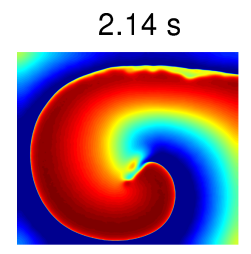

$$
2.8 \mathrm{~s}
$$
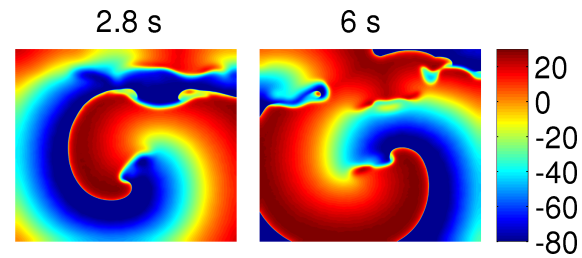

Figure 4. Pseudocolor plot of $\mathrm{V}_{\mathrm{m}}$ showing the spatiotemporal evolution of the break-up of a spiral in the presence of GFD with $p_{f}$ going from $10 \%-100 \%$, and $\mathrm{E}_{\mathrm{f}}=-25 \mathrm{mV}$. The spiral arm breaks up in the upper region, which is the low- $\omega$ region.

As the instability of the spiral waves arises because of the spatial variation of $\omega$ in the medium, the degree of instability of the spiral waves depends on the magnitude of the gradient in $\omega$ that is induced by GFD. We, therefore, study the factors that modulate the variation of $\omega$ with $p_{f}$. We find that high values of $E_{f}$ induce high variation of $\omega$ with $p_{f}$. Figure 3 (a) shows that the variation of $\omega$ with $p_{f}$ is more drastic for $E_{f}=-15 \mathrm{mV}$ (black), as compared to $E_{f}=-25 \mathrm{mV}$ (blue). Moreover, we also find that the variation of $\omega$ with $p_{f}$ is enhanced if we couple more fibroblasts number $N_{f}$ to a myocyte. Figure 3 (b) shows the variation of $\omega$ with $p_{f}$ for three different values of $N_{f}: N_{f}=1$ (black), $N_{f}=2$ (blue), and $N_{f}=3$ (red). We see that variation of $\omega$ increases as we go from $N_{f}=1$ to $N_{f}=3$. These results tells us that, for a given GFD, a spiral wave is more vulnerable to break-up for high values of $E_{f}$ and $N_{f}$ as compared to the low values of $E_{f}$ and $N_{f}$.

\subsection{Spontaneous initiation of spiral waves}

The presence of GFD in the medium can also initiate spiral wave spontaneously if we pace the medium at a high frequency. Figure 5 shows the initiation of spiral waves in a medium with GFD with $p_{f}$ going from $10 \%-100 \%$. We apply periodic line stimuli at the bottom edge of the domain at a pacing cycle length $\mathrm{PCL}=250 \mathrm{~ms}$. The reason for the spiral-wave initiation is as follows. The top region of the medium, because it has a large APD, repolarizes slowly. Now, if we pace the medium, at high-frequency, there is a prominent waveback-wavefront interactions of the waves in the top region. This waveback-wavfront interactions induce corrugations in the wavefronts of the waves (because of the random distribution of fibroblasts), which eventually leads to the initiation of spiral waves.
$3.26 \mathrm{~s}$

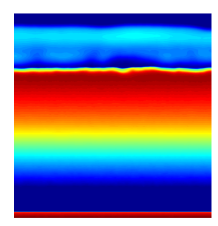

$5.36 \mathrm{~s}$

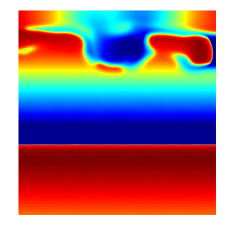

$6.34 \mathrm{~s}$

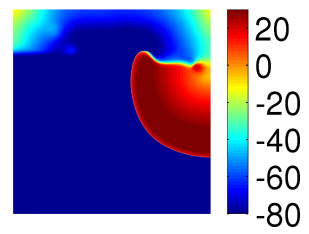

Figure 5. Pseudocolor plot of $\mathrm{V}_{\mathrm{m}}$ showing the the spontaneous initiation of a spiral wave, via high-frequency pacing, in a medium with GFD as shown in fig. 2 (b). The pacing stimuli are applied at the bottom of the domain at PCL $=250 \mathrm{~ms}$.

Such pacing-induced spiral waves occur at highfrequency pacing and not at low-frequency pacing, because the wavefront-waveback interaction between the consecutive waves is more prominent at high-frequency pacing. Figure 6 shows a stability diagram in the $E_{F}-P C L$ plane indicating the regions where spiral waves (magenta color) occur and no spiral waves (black color) occur.

In conclusion, we have shown how fibroblast-myocyte (FM) coupling can affect the the electrical-wave properties in a tissue. We see that FM coupling changed the APD of the myocytes, and this, in turn, modulated the properties of the spiral waves in a tissue, like its frequency $\omega$. We show that $\omega$ decreases with the increase of the percentage of fibroblasts $p_{f}$, because the FM coupling increases the APD of the constituent myocytes. We then study how the gradient in the fibroblast density (GFD) affects the dynam- 


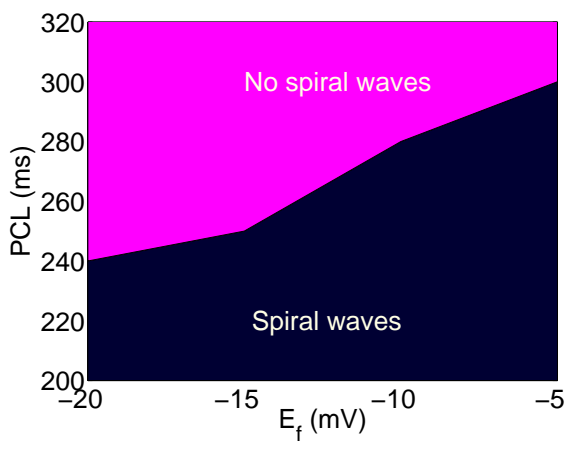

Figure 6. Stability diagram in the $\mathrm{E}_{\mathrm{f}}$-PCL plane. Figure showing the regions where we observe spiral waves (black color) and no spiral waves (magenta color) in the $\mathrm{E}_{\mathrm{f}}-\mathrm{PCL}$ plane.

ics of the spiral waves. We find that GFD induces spiralwave instability in the medium. We show that this instability arises because of the spatial variation of the local value of $\omega$ in the medium induced by the GFD, and the spiral wave breaks in the low- $\omega$ region. Our finding of spiral-wave break in the low- $\omega$ is consistent with the experimental results of Campbell, et al., in monolayers of neonatal-rat ventricular myocytes, where the gradeint in $\omega$ is induced by varying the $\mathrm{I}_{\mathrm{Kr}}$ ion-channel density. We also investigate the factors that affect the variation of $\omega$ with $p_{f}$. We find that the variation of $\omega$ with $p_{f}$ is more for high values of $E_{f}$ and $N_{f}$ as compared to when their values are low. And thus, for a given GFD, the degree of spiral-wave instability is higher at high values of $E_{f}$ and $N_{f}$ as compared to their low values. Finally, we show how GFD in the medium can initiate spiral waves spontaneously via highfrequency pacing. We hope our results will lead to detailed studies of GFD-induced spiral-wave instability at least in in vitro experiments on cell-cultures. At the simplest level, we suggest fibroblast analogs of the experiments of Campbell, etal. [12].

\section{Acknowledgements}

We thank the Department of Science and Technology (DST), India, and the Council for Scientific and Industrial Research (CSIR), India, for financial support, and the Supercomputer Education and Research Centre (SERC, IISc) for computational resources.

\section{References}

[1] Fenton FH, Cherry EM, Hastings HM, Evans SJ. Multiple mechanisms of spiral wave breakup in a model of cardiac electrical activity. Chaos An Interdisciplinary Journal of Nonlinear Science 2002;12(3):852-892.

[2] Efimov IR, Sidorov V, Cheng Y, Wollenzier B. Evidence of three-dimensional scroll waves with ribbon-shaped filament as a mechanism of ventricular tachycardia in the isolated rabbit heart. Journal of cardiovascular electrophysiology 1999;10(11):1452-1462.

[3] Bayly P, KenKnight B, Rogers J, Johnson E, Ideker R, Smith W. Spatial organization, predictability, and determinism in ventricular fibrillation. Chaos An Interdisciplinary Journal of Nonlinear Science 1998;8(1):103-115.

[4] Manabe I, Shindo T, Nagai R. Gene expression in fibroblasts and fibrosis involvement in cardiac hypertrophy. Circulation research 2002;91(12):1103-1113.

[5] Nguyen TP, Xie Y, Garfinkel A, Qu Z, Weiss JN. Arrhythmogenic consequences of myofibroblast-myocyte coupling. Cardiovascular research 2012;93(2):242-251.

[6] Majumder R, Nayak AR, Pandit R. Nonequilibrium arrhythmic states and transitions in a mathematical model for diffuse fibrosis in human cardiac tissue. PLoS one 2012; 7(10):e45040.

[7] Greisas A, Zlochiver S. Modulation of spiral-wave dynamics and spontaneous activity in a fibroblast/myocyte heterocellular tissue - a computational study. IEEE Transactions on Biomedical Engineering 2012;59(5):1398-1407.

[8] Ashikaga H, Arevalo H, Vadakkumpadan F, Blake RC, Bayer JD, Nazarian S, Zviman MM, Tandri H, Berger RD, Calkins $\mathrm{H}$, et al. Feasibility of image-based simulation to estimate ablation target in human ventricular arrhythmia. Heart Rhythm 2013;10(8):1109-1116.

[9] O'Hara T, Virág L, Varró A, Rudy Y. Simulation of the undiseased human cardiac ventricular action potential: model formulation and experimental validation. PLoS Comput Biol 2011;7(5):e1002061.

[10] MacCannell KA, Bazzazi H, Chilton L, Shibukawa Y, Clark RB, Giles WR. A mathematical model of electrotonic interactions between ventricular myocytes and fibroblasts. Biophysical journal 2007;92(11):4121-4132.

[11] Cherry EM, Fenton FH. Suppression of alternans and conduction blocks despite steep apd restitution: electrotonic, memory, and conduction velocity restitution effects. American Journal of Physiology Heart and Circulatory Physiology 2004;286(6):H2332-H2341.

[12] Campbell K, Calvo CJ, Mironov S, Herron T, Berenfeld O, Jalife J. Spatial gradients in action potential duration created by regional magnetofection of herg are a substrate for wavebreak and turbulent propagation in cardiomyocyte monolayers. The Journal of physiology 2012; 590(24):6363-6379.

Address for correspondence:

Soling Zimik

Centre for Condensed Matter Theory, Department of Physics, Indian Institute of Science, Bangalore, 560012, India

solyzk@gmail.com 